\title{
Genome interpreter vies for place in clinical market
}

\section{Launch of system that keeps data local aims to address privacy fears.}

\section{BY MONYA BAKER}

A s DNA sequencing gets faster and cheaper, clinicians are clamouring to use it. A test for malfunctioning genes might show how to treat a tumour or help to diagnose the underlying causes of a disease. But sequencing data are too complex for most clinicians to analyse, and medical institutions are wary of transferring patient data to specialists elsewhere for analysis.

A genome-interpretation company is now offering its solution: a 1-metre-tall, 275-kilogram black box that carries enough storage and processing power to analyse one genome every day, picking out mutations with potential links to disease - in theory, fast enough to inform treatment. But for some, the most important feature of the US $\$ 125,000$ unit is that it is a selfcontained object. In an era of cloud computing and global networks, a machine that keeps its information stubbornly local has growing appeal. "There is a tremendous worry about privacy with sharing patient data," says Martin Tolar, chief executive of Knome, the company in Cambridge, Massachusetts, that produces the device. "The institutions we approached said, 'We want to keep the system within our four walls."

Unveiled on 27 September, the Knome system, knoSYS 100, belongs to an emerging class of services and tools to help clinical researchers to catch up with advances in genome sequencing. That capability "has made itself available faster than we are prepared to deal with", says Vincent Funari, director of the genomics core facility at Cedars-Sinai Medical Center in Los Angeles, California.

The core of Knome's system is not hardware, but software. The machine combs through a newly sequenced genome to find places where humans vary, and annotates them with existing knowledge. This process, known as genome interpretation, can winnow down the millions of variants found in any individual's genome to a handful that might explain a disease (see 'Super sifter'). “Our goal is to take these data and say, 'For this group of patients, these are the 5-10 variants that are most likely to be implicated," explains Tolar. For now, the software is

\section{$\rightarrow$ NATURE.COM} Read more about whole genomes in the clinic at: go.nature.com/yv7rls

\section{SUPER SIFTER}

Genome-interpreting tools work by discarding genetic differences that look inconsequential or lack information, and focusing on a handful likely to be linked to disease. (Schematic is simplified; tools vary.)

\begin{tabular}{|c|c|}
\hline INPUT: Sequenced genome ( 6 billion base pairs) & Variants identified \\
\hline Compare with reference genome and known variation & $\begin{array}{l}3 \text { million to } \\
5 \text { million variants }\end{array}$ \\
\hline Filter for variants in genes & $>20,000$ variants \\
\hline $\begin{array}{l}\text { Compare with genetic databases and genomes of other ill or healthy } \\
\text { people to select rare variants and those associated with disease }\end{array}$ & $\sim 1,000$ variants \\
\hline $\begin{array}{l}\text { If no known causative variant found, run through algorithms to } \\
\text { predict variants likely to disrupt gene function }\end{array}$ & $\sim 100$ variants \\
\hline Use existing knowledge to find variants most likely to cause disease & $\sim 10$ variants \\
\hline OUTPUT: For follow-up analysis & $\sim 10$ variants \\
\hline
\end{tabular}

not meant for clinical diagnosis or medical advice. Clients include drug companies and medical centres researching how to use sequencing for clinical decisions.

Experts warn that genome interpretation is far from mature, and that its reliability depends on the quality of the sequences it analyses. Not all types of variants can be detected, and errors occur at every step before and during interpretation: in the sequencing of fragments of DNA; in matching those millions of fragments with their equivalents in the reference genome; and in detecting differences. "The interpretation of variants is absolutely dependent on accurate variant identification," says Karl Voelkerding, medical director for genomics and bioinformatics at ARUP Laboratories, an assay facility in Salt Lake City, Utah.

Then there is the challenge of working out which detected variants are relevant to disease. The protocols are imperfect and the various annotation tools access different data in different ways and so supply a variety of answers. And all annotation tools uncover many 'variants of unknown significance, about which too little is known to assess whether or not they affect a person's health. Any variant that might be used to provide a diagnosis or guide patient care must be verified independently by separate experiments.

But just organizing information into a useful form is a big step forward, says George Church, a genomicist at Harvard Medical School in Boston, Massachusetts, and co-founder of Knome. The process, he says, "is not about perfection. It's about delivering a high-quality interpretation based on current knowledge."

Knome's device may be well placed to tap a nascent clinical market in which data is preferentially kept on site, but sequencing companies are also making inroads with software that requires genomic data to be transferred elsewhere. For example, Illumina, based in San Diego, California, offers free data storage and variant identification for clients who upload sequencing data to its cloud-computing platform, which has an open programming interface. Illumina has contracts with a suite of other companies to develop data-analytic applications in the cloud. As more centres begin using sequencing data, it is expected that policies and procedures for using the cloud will mature.

David Goldstein, a genomicist at Duke Institute for Genome Sciences and Policy in Durham, North Carolina, says that although commercial genome interpreters may be valuable in the clinic, he doubts that they will make headway in the larger genomics research centres. Commercial offerings rely on standard references and techniques that can make their approach too constraining for genomics research, he says. "We've never had a 12-month stretch where we've done something the same way."

But for clinicians interested more in working out how best to apply current genomics knowledge, prepackaged software and hardware may be just the ticket. "Last year we had zero clinical institutes" as clients, says Tolar, "and this year we have 20." - SEE EDITORIAL P.143 